\title{
Vorwort zur zweiten Auflage
}

Die Neuauflage vertieft die erstmals 1999 gezogene Bilanz der Strafverfolgung von DDR-Unrecht. Einbezogen sind alle seit dem Erscheinen der ersten Auflage ergangenen Entscheidungen sowie neuere kritische Stellungnahmen. Zudem wurden die in der Zwischenzeit erschienene Reihe Marxen/Werle (Hrsg.), „Strafjustiz und DDR-Unrecht. Dokumentation“ sowie die Broschüre Marxen/Werle/ Schäfter, „Die Strafverfolgung von DDR-Unrecht. Fakten und Zahlen“ eingearbeitet, die, wie schon die 1. Auflage, aus dem Forschungsprojekt „Strafjustiz und DDR-Vergangenheit“ hervorgegangen sind.

Für die Neuauflage wurde der Text vollständig überarbeitet. Die Einbeziehung der nach 1999 ergangenen Entscheidungen machte durchgängig eine Aktualisierung des Zahlenmaterials erforderlich.

30 Jahre nach dem Mauerfall liegt damit eine abgeschlossene Gesamtdarstellung der strafrechtlichen Aufarbeitung des DDR-Unrechts vor. Sie schildert die Rahmenbedingungen der Aufarbeitung, stellt die Fallgruppen des DDRUnrechts vor und informiert auf der Grundlage zuverlässiger Zahlen über Verfahren und Ergebnisse. Darüber hinaus werden die Grundlinien der strafjuristischen Aufarbeitung herausgestellt und kritisch bewertet - auch in international und historisch vergleichender Perspektive.

Für wertvolle Unterstützung bei der Fertigstellung des Manuskripts danken wir den Mitarbeiterinnen und Mitarbeitern sowie den Promovierenden des Lehrstuhls von Moritz Vormbaum. Dr. Kathrin Fenrich hat wichtige Beiträge zur Überarbeitung des ersten Teils geleistet. Am Abschnitt über die Gewalttaten an der deutsch-deutschen Grenze hat Tobias Köpcke auch durch Recherchetätigkeiten mitgewirkt. Neuere Informationen zum Doping im DDR-Leistungssport verdanken wir Michaela Galandi. Leonie Brinkmann hat umfangreiche redaktionelle Arbeiten an den Fußnoten und Verzeichnissen durchgeführt.

Dem Verlag de Gruyter danken wir für die Bereitschaft, die zweite Auflage des Bilanzbandes zu veröffentlichen. Lili Hammler, Project Editor von de Gruyter, danken wir für die angenehme Zusammenarbeit und für ihre Geduld.

Berlin und Münster, April 2020

Klaus Marxen, Gerhard Werle, Moritz Vormbaum 